\title{
A Review Of Design And Control Of Automated Guided Vehicle Systems
}

\author{
Tuan Le-Anh and M.B.M. De Koster
}

\begin{tabular}{|l|l|}
\hline \multicolumn{2}{|l|}{ ERIM REPORT SERIES RESEARCH IN MANAGEMENT } \\
\hline ERIM Report Series reference number & ERS-2004-030-LIS \\
\hline Publication & May 2004 \\
\hline Number of pages & 34 \\
\hline Email address corresponding author & Ituan@fbk.eur.nl \\
\hline Address & Erasmus Research Institute of Management (ERIM) \\
& Rotterdam School of Management / Rotterdam School of \\
& Economics \\
& Erasmus Universiteit Rotterdam \\
& P.O. Box 1738 \\
& 3000 DR Rotterdam, The Netherlands \\
& Phone: +31 10 408 1182 \\
& Fax: $\quad+31104089640$ \\
& Email: info@erim.eur.nl \\
& Internet: www.erim.eur.nl \\
\hline
\end{tabular}

Bibliographic data and classifications of all the ERIM reports are also available on the ERIM website: www.erim.eur.nl 


\title{
ERASMUS RESEARCH INSTITUTE OF MANAGEMENT
}

\author{
REPORT SERIES \\ RESEARCH IN MANAGEMENT
}

\begin{tabular}{|c|c|c|}
\hline \multicolumn{3}{|c|}{ BIBLIOGRAPHIC DATA AND CLASSIFICATIONS } \\
\hline Abstract & \multicolumn{2}{|c|}{$\begin{array}{l}\text { This paper presents a review on design and control of automated guided vehicle systems. We } \\
\text { address most key related issues including guide-path design, estimating the number of } \\
\text { vehicles, vehicle scheduling, idle-vehicle positioning, battery management, vehicle routing, and } \\
\text { conflict resolution. We discuss and classify important models and results from key publications in } \\
\text { literature on automated guided vehicle systems, including often-neglected areas, such as idle- } \\
\text { vehicle positioning and battery management. In addition, we propose a decision framework for } \\
\text { design and implementation of automated guided vehicle systems, and suggest some fruitful } \\
\text { research directions. }\end{array}$} \\
\hline \multirow{3}{*}{$\begin{array}{l}\text { Library of Congress } \\
\text { Classification } \\
\text { (LCC) }\end{array}$} & $5001-6182$ & Business \\
\hline & $5201-5982$ & Business Science \\
\hline & HE 383 & $\begin{array}{l}\text { Transportation - waterways in relation to other forms of } \\
\text { transportation }\end{array}$ \\
\hline \multirow{3}{*}{$\begin{array}{l}\text { Journal of Economic } \\
\text { Literature } \\
\text { (JEL) }\end{array}$} & M & Business Administration and Business Economics \\
\hline & $\begin{array}{l}\text { M } 11 \\
\text { R } 4\end{array}$ & $\begin{array}{l}\text { Production Management } \\
\text { Transportation Systems }\end{array}$ \\
\hline & R 4 & Transportation Systems \\
\hline \multirow{3}{*}{$\begin{array}{l}\text { European Business Schools } \\
\text { Library Group } \\
\text { (EBSLG) }\end{array}$} & $85 \mathrm{~A}$ & Business General \\
\hline & $\begin{array}{l}260 \mathrm{~K} \\
240 \mathrm{~B}\end{array}$ & $\begin{array}{l}\text { Logistics } \\
\text { Information Systems Management }\end{array}$ \\
\hline & $260 \mathrm{~K}$ & Logistics \\
\hline \multicolumn{3}{|c|}{ Gemeenschappelijke Onderwerpsontsluiting (GOO) } \\
\hline \multirow[t]{3}{*}{ Classification GOO } & 85.00 & Bedrijfskunde, Organisatiekunde: algemeen \\
\hline & $\begin{array}{l}85.34 \\
85.20\end{array}$ & $\begin{array}{l}\text { Logistiek management } \\
\text { Bestuurlijke informatie, informatieverzorging }\end{array}$ \\
\hline & 85.34 & Logistiek management \\
\hline \multirow[t]{3}{*}{ Keywords GOO } & \multicolumn{2}{|c|}{ Bedrijfskunde / Bedrijfseconomie } \\
\hline & \multicolumn{2}{|c|}{ Bedrijfsprocessen, logistiek, management informatiesystemen } \\
\hline & \multicolumn{2}{|c|}{ Goederenoverslag; Automated guided vehicles, literatuuroverzichten } \\
\hline Free keywords & \multicolumn{2}{|c|}{ Material handling system; Automated guided vehicle; Literature overview } \\
\hline
\end{tabular}




\title{
A REVIEW OF DESIGN AND CONTROL OF AUTOMATED GUIDED VEHICLE SYSTEMS
}

\author{
Tuan Le-Anh", MBM De Koster \\ Rotterdam School of Management, Erasmus University Rotterdam, P.O. Box 1738, 3000 DR Rotterdam, The Netherlands \\ *Corresponding author. Tel.: +31 10 4082027; fax: + 3110408 9014; e-mail LTuan@fbk.eur.nl
}

\begin{abstract}
This paper presents a review on design and control of automated guided vehicle systems. We address most key related issues including guide-path design, estimating the number of vehicles, vehicle scheduling, idle-vehicle positioning, battery management, vehicle routing, and conflict resolution. We discuss and classify important models and results from key publications in literature on automated guided vehicle systems, including often-neglected areas, such as idle-vehicle positioning and battery management. In addition, we propose a decision framework for design and implementation of automated guided vehicle systems, and suggest some fruitful research directions.
\end{abstract}

Keywords: Material handling system; Automated guided vehicle; Literature overview

\section{Introduction}

Material handling systems using automated guided vehicles (AGVs) are commonly used in facilities such as manufacturing plants, warehouses, distribution centers and terminals. Figure 1 gives an example of such a material handling system in a distribution center of computer hard and software (De Koster et al., 2004), in which guided vehicles transport pallets (loads) between locations, e.g. from receiving lanes to storage areas, and from storage areas to shipping lanes. 


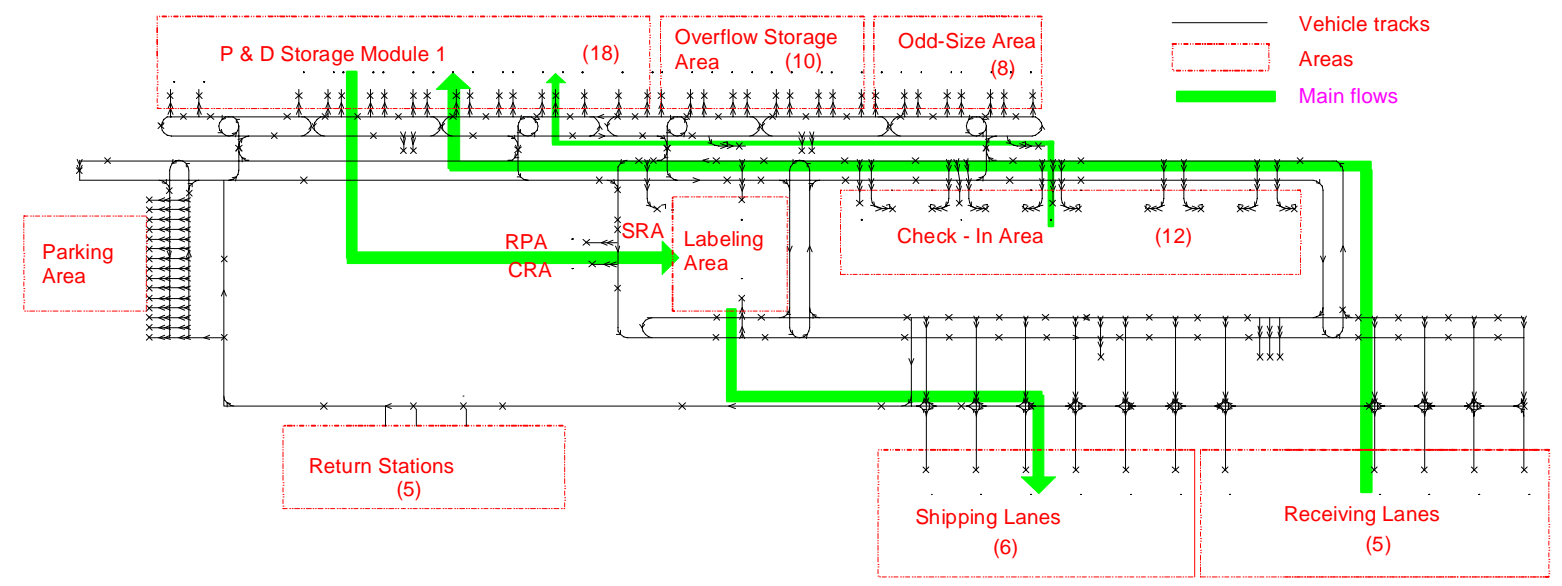

Figure 1 The guided-vehicle system of a distribution center

The performance of the material handling system directly affects the performance of the whole facility. Basically, the relevant issues at facilities using automated guided vehicles can be divided into the following main categories: guide-path design, estimating the required number of vehicles, vehicle scheduling, idle-vehicle positioning, battery management, vehicle routing and conflict resolution. These issues relate to different levels of the decisionmaking process. The guide-path design problem can be seen as a problem at strategic level. The decision at this stage has a strong effect on decisions at other levels. Issues at tactical level include estimating the number of vehicles, scheduling vehicle, positioning idle vehicles and, managing battery-charging scheme. Finally, the vehicle routing and conflict resolution problems are addressed at the operational level. During the design and implementation process, some interactions and iterations can be seen between steps. The type of the guidepath system directly influences the number of vehicles required and the complexity of the vehicle scheduling system. The scheduling system also affects the number of vehicles required.

Modern AGV systems differ from the classic ones as described for instance in the books of Jünemann and Schmidt (2000) and Tompkins et al. (2003) in several respects. Rather than using fixed paths, many modern AGVs are free-ranging, which means their preferred tracks are software programmed, and can be changed relatively easy when new stations or flows are added. A second difference is in the way they can be controlled. Agent technology allows decisions to be taken by these smart vehicles that in the past were taken by central controllers. This leads to adaptive, self-learning systems and is particularly appropriate for large, complex systems with many vehicles and much potential vehicle interference. These developments do not imply that the traditional decision-making problems become obsolete. 
Rather, they lead to new challenges for research. We both discuss the traditional AGV system decision-making problems and the impact of these developments on decision-making.

There are few review papers on AGV systems. However, they concentrate on only limited parts of the problem (Qiu et al. (2002) focus on scheduling and routing problems) or are not up to date (Sinriech, 1995; Co and Tanchoco, 1991; King and Wilson, 1991). Moreover, they ignore some areas such as idle-vehicle positioning and battery management. This paper attempts to fill this gap, by giving an extended overview of existing literature, including the most recent contributions, and also structures the design and implementation decision process for AGV systems. For each area, we review and classify key decision models. In addition, a new classification for dispatching rules, a guideline for selecting a suitable scheduling system and a decision framework for design and implementation of AGV systems are proposed. We also consider idle-vehicle positioning and battery management problem, which many review papers neglect. Finally, we make suggestions for some fruitful future research directions.

We start with an introduction to AGV systems followed by a discussion of guide-path design in section 2. Section 3 investigates the problem of determining the required number of singleor multi-load vehicles. Section 4 reviews the problem of managing an AGV system including online and offline scheduling, vehicle dispatching, idle-vehicle positioning, battery management, vehicle routing and conflict resolution. In section 5, we illustrate the design and implementation decision process of an AGV system by a decision framework. Finally, in section 6 we draw conclusions and identify subjects for further research.

\section{Guide-path design}

Guide-path design is an important problem in AGV system design and is one of the very first problems to be considered. The guide-path depends greatly on the allocation of shop-floor space, layout of storage zones and the arrangement of handling stations. In most cases, the shop-floor space is fixed and it imposes constraints on the guide-path design problem. The vehicle guide-path is usually represented such that aisle intersections, pick-up and delivery (P/D) locations can be considered as nodes on a graph connected by a set of arcs. The arcs describe the paths that vehicles can follow when moving from node to node. Directed arcs between two nodes indicate the direction of the vehicle flow. Cost can be assigned to each arc representing the distance between the two end points of a segment or the time required by a 
vehicle to travel along the arc. This representation can be seen as a network-based system that is useful for formulating the guide-path design problem. The guide-path system can be classified by the characteristics indicated in Table 1 and will be explained further in the next section.

Table 1 Characteristics of guide-path systems

\begin{tabular}{lll}
\hline Flow topology & Number of parallel lanes & Flow direction \\
\hline Conventional & Single lane & Unidirectional flow \\
Single-loop & Multiple lanes & Bidirectional flow \\
Tandem & & \\
\hline
\end{tabular}

The first strategically important issue here is selecting the performance criteria for designing a guide-path system.

\subsection{Performance criteria}

An important question is which performance criteria are most important for the guide-path design problem. Beamon (1998) describes several important performance criteria for automated guided vehicle systems such as vehicle travel time, vehicle utilization, queue length, and material handling cost. The most common performance criterion in guide-path design is minimizing the total vehicle travel distance corresponding to a given layout and flows (Gaskins and Tanchoco, 1987; Kaspi and Tanchoco, 1990). Kaspi et al. (2002) include both the vehicle loaded and empty travel time in the objective function. Lim et al. (2002) use the total vehicle travel time (including the loaded and empty vehicle travel and waiting time caused by congestion or vehicle interferences) as the objective function. Several authors use multiple objectives. Kim and Tanchoco (1993) consider the travel cost and the cost of each path segment. Chen et al. (1999) use the total vehicle travel time and the trip failure rate as performance measures for their model. Talbot (2003) uses the required number of vehicles and the guide-path length to measure the system performance. Generally, multi-criteria objective functions lead to a better solution quality.

The next three sections review the design problem for the three most important guide-path systems in literature. The literature does not clearly indicate when and where a specific type should be selected, but based on the system requirements, characteristics of facilities and of each guide-path system, decision makers can select one of them for their system. 


\subsection{Conventional guide-path system}

A conventional guide-path system is a network of guide-paths connecting all workstations in a system that have transportation requirements. This network may contain junctions, intersections, and shortcuts. The conventional guide-path can be unidirectional or bidirectional. In the unidirectional guide-path system vehicles travel in only one direction of the guide-path. In the bidirectional system, both travel directions are possible.

\section{a Unidirectional guide-path system}

The unidirectional conventional guide-path system is very popular in practice, particularly in warehouses or distribution centers (Figure 1). Several authors (Gaskins and Tanchoco, 1987; Gaskins et al., 1989; Goetz and Egbelu, 1990; Kaspi and Tanchoco, 1990; Sinriech and Tanchoco, 1991; Kim and Tanchoco, 1993) formulate the guide-path design problem as a 0-1 integer-programming (IP) model. These models decide the guide-path sections and their directions that minimize the total loaded vehicle travel distance. The size of the guide-path desogn IP model can be huge for practical problems. To speed up the solution procedure, Goetz and Egbelu (1990) focus on only the major flows between departments and Sinriech and Tanchoco (1991) consider only intersection nodes in their branch-and-bound algorithm.

Ignoring the empty vehicle travel time is the main drawback of above models. Kaspi et al. (2002) propose an improved formulation of the model in Kaspi and Tanchoco (1990) by incorporating the empty vehicle travel in the objective function and reducing the number of binary variables. Their formulation is illustrated below:

$$
\begin{aligned}
& \min \sum_{i j \mid m} L_{i j l m} d_{i j}+\sum_{i j l m} E_{i j / m} d_{i j} \\
& L_{i j m} \leq M Z_{i j} \forall i, j \text { and } \forall l, m \text { (1) } \\
& Z_{i j}+Z_{j i} \leq 1 \forall i, j \\
& \sum_{i} Z_{i j} \geq 1 \quad \forall j \\
& \sum_{k} L_{l k l m}=f_{l m} \quad \forall l, m \\
& \sum_{k} L_{k m l m}=f_{l m} \quad \forall l, m \\
& \sum_{k} Z_{j k} \geq 1 \quad \forall j \\
& \sum_{i} L_{i j l m}=\sum_{k} L_{j k l m} \forall l, m \forall j(7) \\
& \begin{array}{l}
E_{i j l m} \leq M Z_{i j} \forall i, j \text { and } \forall l, m \text { (8) } \\
\sum_{j l} E_{m j m l}=\sum_{l} f_{l m} \quad \forall m
\end{array} \\
& \sum_{i m} E_{i l m l}=\sum_{m} f_{l m} \quad \forall l \\
& \sum_{i} E_{i j m l}=\sum_{k} E_{j k m l} \forall l, m \forall j(11)
\end{aligned}
$$

where $N$ - number of entries in the from-to chart; $f_{l m}$ - flow intensity from pick-up node $l$ to delivery node $m$; $d_{i j}$ - length of arc $i-j$ (the distance from node $i$ to an adjacent node $j$ ); $Y_{l m}$ - path length from node $l$ to node $m$; $W_{m l^{-}}$flow intensity of empty vehicles from delivery station $m$ to pick-up station $l ; L_{i j l m}$ - loaded vehicle traffic intensity of $l-m$ delivery path along arc $i-j ; E_{i j l m}$ - empty vehicle traffic intensity of $m$ - $l$ delivery path along arc $i-j ; Z_{i j}=1$ if arc $i-j$ is directed from node $i$ to node $j$, = 0 otherwise; $M$ - a large number. 
The objective function is to minimize both the loaded and empty vehicle travel distance. The constraint set (1-4) ensures the connectivity of the network-flow. The constraint sets (5-7) and (8-11) ensures the flow feasibility of the network-flow. This is a mixed-integer (0-1) programming model, which is difficult to solve.

They solve this model using a branch-and-bound depth-first search algorithm. The empty vehicle flow $\left(W_{m l}\right)$ is computed during the execution of the search algorithm by solving a transportation problem to distribute empty vehicles from the group of delivery stations to the group of pick-up stations to minimize the total distribution flow.

Besides empty vehicle travel time, time lost caused by vehicle interference also impacts the quality of solutions. Lim et al. (2002) consider total vehicle travel, including the empty and loaded vehicle travel time and time lost caused by congestion and vehicle interference in the design model. They estimate the total vehicle travel time using the Q-learning technique (a process of learning how to match states with actions in order to maximize a numerical reward). They show that their results are superior to those of Kim and Tanchoco (1993). Obviously, the quality of their solution depends heavily on the accuracy of the travel time computed by the Q-learning process.

A more complicated problem is tackled by Johnson and Brandeau (1993, 1994); Al-Sultan and Bozer (1998). Their models select path configuration and the P/D stations at a same time. Al-Sultan and Bozer (1998) use a simulated annealing heuristic to solve the guide-path design problem. In their paper, they also note that the model of Gaskins and Tanchoco (1987) may generate infeasible or non-optimal solutions. Johnson and Brandeau $(1993,1994)$ use the

benefit of AGV systems and fixed cost of setting a pick-up/delivery (P/D) station as the objective function instead of total vehicle travel distances. Johnson and Brandeau (1993)'s model also determines the number of vehicles required to warrant a service level (expected time until a workstation is replenished from the central depot). The pool of vehicles is approximated by an M/G/c queuing system. They formulate the problem as a 0-1 IP model and solve it using a branch-and-bound algorithm.

\section{Bidirectional guide-path system}

The conventional bidirectional guide-path is not popular in material handling systems, although it can result in a higher productivity than the corresponding unidirectional guidepath system. The main reason is that the control problem in such systems becomes very complicated. This problem can be resolved by using dual unidirectional lanes. However, the 
dual lanes system needs more space and is more costly. In literature, there are only few studies on the conventional bidirectional guide-path system (Egbelu and Tanchoco, 1986; Gaskins et al., 1989). Egbelu and Tanchoco (1986) provide a guideline for design of singlelane bidirectional guide-path systems. Gaskins et al. (1989) propose a model for a bidirectional guide-path system in which the travel distance and the number of lanes are minimized. The number and direction of lanes are decided in their model. The capacity of each lane and the maximum number of parallel lanes are given and fixed.

Bidirectional guide-path systems are particularly used in systems where vehicle interference rarely happens rarely such as in tandem guide-path systems (section 2.4).

\subsection{Single-loop guide-path system}

The main difference between the single-loop and the conventional guide-path system is that in the single-loop layout, vehicles travel in only one loop without any shortcut or alternative routes (e.g. the loop in the left part of Figure 2). The travel mode in the single-loop system is usually unidirectional. Bidirectional traveling is possible but in this case vehicle interference is likely to happen. Vehicles in single-loop systems can be controlled by simple dispatching rules such as first-encountered-first-serve (FEFS), implying that an empty vehicle should pick up the first load it encounters. Tanchoco and Sinriech (1992) propose an optimal procedure to design a single-loop system. This procedure includes five components:

(1) An IP formulation is used to find an initial valid loop (a valid single loop problem - VSLP) - a valid loop contains at least one segment for each department in the facility layout.

(2) A procedure (find all single loops - FASL) enumerates all possible valid single-loop guide paths using a two-phase approach. The first phase creates new valid single loops by expanding the initial loop. The second phase generates more valid loops by contracting the last loop in the previous phase.

(3) Loop-elimination rules are used to reduce the numbers of candidate loops (inferior loops are eliminated).

(4) A model determines locations of pick-up and delivery stations for each department along a singleloop path, by solving a mixed integer programming (MIP) problem (a single-loop station location problem - SLSLP). The objective is minimizing the total flow times in the system.

(5) A lower bound calculation procedure computes lower bounds for candidate loops. Instead of solving the SLSLP problem for each valid single loop that is very time-consuming, this lower bound can help to eliminate some inferior loops quickly. 
Their iterative algorithm involving of solving two 0-1 integer-programming (IP) models is very time consuming for realistic problems.

Some other models and solution procedures for the single loop guide-path system design are proposed in Sinriech and Tanchoco (1993), Chen et al. (1999), and Asef-Vaziri et al. (2000). Chen et al. (1999) present a MIP model to design guide-paths for a single-loop dual rail (path) system (SLDR), a special class of the single-loop system. This system contains only one loop (single-loop), but vehicles use two parallel tracks. This model also captures the vehicle failure rate in the objective function, which is claimed to produce more reliable results. This SLDR problem was solved using CPLEX (an optimization package). An instance containing 13 P/D locations needs about 2hrs of computation time using CPLEX on a SPARC station 2. Asef-Vaziri et al. (2000) propose an alternative formulation to Tanchoco and Sinriech (1992) that has a smaller number of binary variables and considers a larger set of feasible integer solutions. Their formulation takes the design of a unidirectional singleloop and the location of P/D stations into account at the same time.

The throughput of the single-loop system drops slightly compared with the throughput of the conventional system (Tanchoco and Sinriech, 1992). To obtain the same throughput with the conventional system, the single-loop system needs more vehicles. Obviously, the single-loop system eliminates the inference problem at intersections (this system has no intersection at all). However, with multiple vehicles operating in the same loop, vehicle interference is still possible, since vehicles may have different operating speeds, etc.

\subsection{Tandem guide-path system}

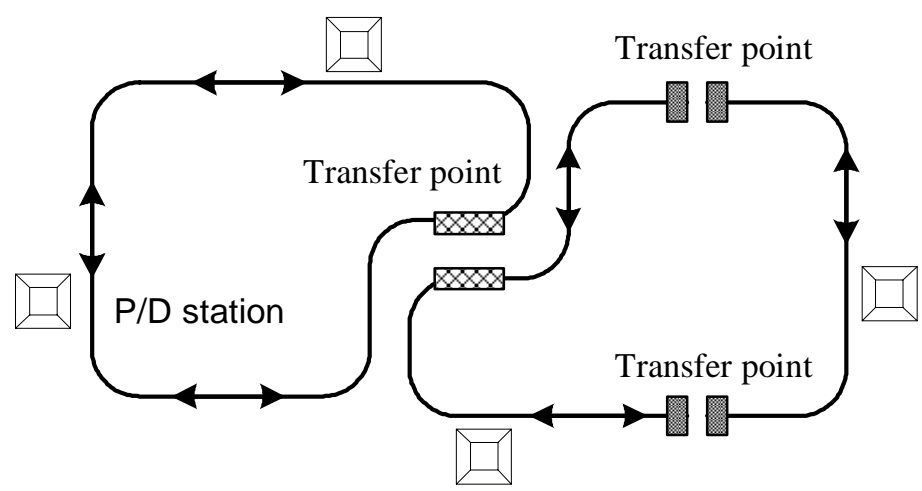

Figure 2 A tandem guide-path system with three zones (one loop and two segments) 
The tandem guide-path system was first introduced by Bozer and Srinivasan (1991). The tandem guide-path system contains multiple zones. Only one vehicle serves each zone and transfer stations are used to interface between zones (Figure 2). In case zones are loops, we have a tandem-loop configuration. A number of non-overlapping single-loop paths provide transportation possibilities. In tandem guide-path systems, a job may require more than one vehicle to transport it to its destination. Vehicle blocking and interference problems are totally eliminated. Bozer and Srinivasan (1991) also propose an analytical model to study the throughput of a single vehicle loop.

Bozer and Srinivasan (1992) introduce an algorithm based on a set-partitioning approach to decompose a system into non-overlapping, single-vehicle zones operating in tandem. The procedure starts by generating promising subsets of workstations. A vehicle must have enough capacity to serve a subset (with workload $\omega$ ). After that, the feasibility of each subset is checked. These subsets (columns) are fed into a set-partitioning model, which is used to identify the best tandem layout. The set-partitioning model is given below:

$$
\begin{aligned}
& \text { minimize } \quad Z \\
& \text { subject to: } \quad z-\omega_{p} x_{p} \geq 0 \quad \forall p \quad \text { (1) } \quad \sum_{p} a_{i p} x_{p}=1 \quad \forall i \quad \text { (2) } \quad \sum_{p} x_{p}=L
\end{aligned}
$$

The first constraint ensures the workload in any zone does not exceed $z$ (maximum workload). The second constraint ensures that each workstation is assigned to only one column (zone) and the last constraint forces the resulting partition to have exactly $L$ zones. The objective of the set-partitioning problem is to avoid generating bottleneck zones by evenly distributed the overall workload among the zones as much as possible. This model was solved using LINDO, however to solve big problems, a more efficient algorithm is proposed. In Bozer and Srinivasan (1992), a vehicle in the tandem guide-path system does not need to use FEFS, but it can use other dispatching rules as well.

Huang (1997) introduces a variation of tandem configurations with an additional transportation center. This particular tandem configuration serves transportation jobs quicker and needs smaller number transfer stations between loops. However, the transportation center needs more space and requires higher investment. His procedure allocates a transfer point in each zone and constructs a transportation center to connect them for a given tandem system. Yu and Egbelu (2001) introduce a variable-path tandem system based on partitioning a 
conventional guide-path into non-overlapping tandem zones. A dedicated vehicle serves each partition (sub-network) and additional transfer points provide interfaces between adjacent networks. Each sub-network is not necessarily a loop, so the dedicated vehicle has a greater flexibility in routing.

Ross et al. (1996) compare the performance (AGV utilization, mean flow time, mean tardiness, mean percent tardy) of the tandem and conventional systems for specific configurations. They show that the tandem system performs as efficient as the conventional system. In comparison with the conventional system, the tandem system is simpler to control, has no congestion problem and is easier to expand. However, the tandem system requires additional transfer buffers which are costly and increase the handling time. This disadvantage may reduce the system throughput. The tandem system also has other disadvantages such as less tolerance to system failures.

\section{․ The segmented guide-path system (segmented flow topology - SFT)}

Sinriech and Tanchoco (1995) describe a specific type of guide-path systems: the segmented flow topology (Figure 2). The SFT system contains one or more zones, each of which is separated into non-overlapping segments served by a single vehicle. Transfer buffers are situated at both ends of each segment and serve as interface devices between the segments (Figure 2). A SFT system may be not fully connected, depending on logical material flow requirements. Considering the SFT system carefully, it appears to be very similar to a general tandem system. Even in the case when the SFT system is not fully connected, we may interpret it as a combination of several tandem sub-systems. Sinriech and Tanchoco (1995) propose a procedure to solve the segmented flow-path layout design problem. According to their results, the SFT system outperforms the conventional system in many criteria. However, even a small problem requires a large amount of time to solve. In Sinriech and Tanchoco (1997), a more efficient procedure was developed for the SFT design problem, which can be implemented for real-life problems. The main disadvantage of the SFT system is that it requires additional transfer stations.

To summarize this guide-path system section we describe some characteristics of guide-path systems in Table 2 and Table 3. 
Table 2 A comparison of guide-path systems

\begin{tabular}{|c|c|c|c|}
\hline Features & Conventional & Single-loop & Tandem \\
\hline $\begin{array}{l}\text { Number of mutually exclusive } \\
\text { zones }\end{array}$ & $\begin{array}{l}\text { One zone, fully } \\
\text { connected system }\end{array}$ & $\begin{array}{l}\text { One zone, fully } \\
\text { connected system }\end{array}$ & $\begin{array}{l}\text { Split system which retains } \\
\text { connectivity through transfer } \\
\text { buffers, can be non-connected } \\
\text { for a special case (SFT) }\end{array}$ \\
\hline Number of vehicles per zones & Multiple & Multiple & Single \\
\hline $\begin{array}{l}\text { Operating with a bidirectional } \\
\text { system }\end{array}$ & Difficult & Difficult & Simple \\
\hline Traffic control & Difficult & Easy & Easy \\
\hline Vehicle scheduling/ dispatching & $\begin{array}{l}\text { Complex scheduling/ } \\
\text { dispatching system }\end{array}$ & Simple & Simple \\
\hline Congestion (probability) & High & Low & No \\
\hline $\begin{array}{l}\text { Intermediate buffers required } \\
\text { (to transfer loads between loops } \\
\text { or transfer points) }\end{array}$ & No & No & Yes \\
\hline
\end{tabular}

Table 3 Advantages and disadvantages of guide-path systems

\begin{tabular}{cll}
\hline $\begin{array}{c}\text { Guide-path } \\
\text { system }\end{array}$ & \multicolumn{1}{c}{ Advantages } & \multicolumn{1}{c}{ Disadvantages } \\
\hline \multirow{3}{*}{ Conventional } & - Flexible in routing & - Efficiency achieved by utilizing \\
& $\begin{array}{l}\text { alternative routes } \\
\text { - Shorter travel distances }\end{array}$ & - Complicated to control \\
& - Tolerance to system failures & $\begin{array}{l}\text { - Congestion, interference problems are likely to } \\
\text { - Difficulty of expansion }\end{array}$ \\
\hline \multirow{4}{*}{ Single-loop } & - Simplicity of control & - Less flexible in routing \\
& - Congestion, blocking, interference & - Less tolerance to system failures \\
& problems are reduced in comparison & - Vehicle blocking and interference are possible \\
& with the conventional system & - Extra transport capacity needed \\
& & - Longer travel for loads \\
\hline \multirow{3}{*}{ Tandem } & - No vehicle congestion and & - Difficulty of expansion \\
\hline & interference & - Additional transfer buffers are required \\
& - Simplicity to control & - Restriction of one vehicle per zone \\
& - Easy expansion & - Less tolerance to system failures \\
& - Effective use of the bidirectional path & - Some loads are handled by more than one vehicles \\
& & - Additional time is required to transfer loads at \\
\end{tabular}

Many modern AGV systems do not use fixed guide-paths (induction tracks). The guide-paths may, for example, be computer-programmed and uploaded to the vehicles' controllers. These vehicles are free-ranging and find their way using optical (laser), magnetic, odometer, gyroscope, vision, or radio- frequency techniques (Tompkins et al., 2003). In order to make full use of the flexibility capabilities of such systems, smart AGVs are needed. The flexibility of changing guide-paths demands a capability to adapt the guide-path system satisfying new system requirements. In this case, obtaining the optimal guide-path system as a first objective becomes less important, however the system's flexibility becomes crucial.

An emerging problem in the guide-path design is selecting an appropriate type of guide-path system, but a guideline to select a suitable guide-path system is not available yet. The 
conventional guide-path system can be seen regularly in warehouses and distribution centers (De Koster et al., 2004), the single-loop system is used, for example, in cross-dock centers. The tandem configuration may be more appropriate for manufacturing environments where workstations are grouped into manufacturing cells.

\section{Estimating the number of vehicles}

\subsection{Single-load capacity vehicles}

The number of vehicles heavily influences the performance of AGV systems (Van der Meer, 2000). AGVs are usually expensive, so determining types and the appropriate number of vehicles is important. For a tandem configuration, the required number of vehicles is equal to the number of zones, but for other guide-path systems this number has to be estimated. According to Egbelu (1987) there are three main factors affecting the required number of vehicles: (1) the guide path layout, (2) location of load transfer points and (3) vehicle dispatching strategies. He proposes four analytical models to calculate the number of vehicles based on several information sources such as the expected number of loaded trips between stations and the number of workstations in the facility. Using one of these models (model 4) Egbelu (1987) estimates the number of vehicles as:

$$
N=\left[\left(\sum_{i=1}^{n} \sum_{j=1}^{n} D_{i j} / V\right)+\left(\sum_{i=1}^{n} \sum_{j=1}^{n} f_{i j}\right) \times\left(t_{u}+t_{l}\right)\right] /(60 T-t)
$$

with $n$ - number of workstations; $f_{i j^{-}}$expected number of loaded trips required between workstation $i$ and workstation $j$ during the period or shift; $D_{i j}$ - the estimated empty and loaded travel distance between stations $i$ and $j$; $T$ - length of the period of shift during which the $f_{i j}$ exchange occur; $V$ - average vehicle travel speed; $t_{l^{-}}$ mean time to load a vehicle; $t_{u}$ - mean time to unload a vehicle; $t$ - expected lost time by each vehicle during a time period of $T$ due to battery change.

Egbelu (1987) indicates that the first three models in his paper are normally over optimistic estimating the number of vehicles. The fourth model (described above) provides a reasonably good estimation in most cases except when the shortest-travel-distance first vehicle dispatching rule is used. The main factors that lead to difference results between models is the method of estimating the empty travel and time lost caused by blocking. Actually, Egbelu (1987) mentions the important role of dispatching rules in estimating the required number of vehicles, but he does not take it into account explicitly. Similar approaches are proposed by Maxwell and Muckstadt (1982), Mahadevan and Narendran (1993). 
Since the dispatching rule has an important role in estimating the required number of vehicles, methods explicitly considering dispatching rules in the estimation model normally provide better results. Srinivasan et al. (1994) analyze a system using only one vehicle traveling under the modified-FCFS rule. They extend the result for the multi-vehicle case, by approximating K-vehicles by a single vehicle traveling K-times faster. They gain a good approximation for vehicle statistics such as the fraction of time a vehicle travels empty and also for the required number of vehicles. The quality of their model deteriorates when the system requires a large number of vehicles. Shen and Kobza (1998) propose another analytical model to estimate the number of vehicles in light-traffic systems.

Other analytical approaches estimating the required number of vehicles include: queuing models (Tanchoco et al., 1987; Talbot, 2003); statistical approach (Arifin and Egbelu, 2000); multi-criteria decision modeling (Sinriech and Tanchoco, 1992a) and network-flow modeling (Vis et al., 2001). The queuing model of Talbot (2003) estimates the number of vehicles to achieve a desired fill rate (the probability to have a vehicle available at a station to satisfy a request). The quality of estimation deteriorates in light-traffic systems, as it tends to overestimate the number of vehicles. Vis et al. (2001) propose a network-flow formulation for determining the number of AGVs required at a semi-automated container terminal in which a job is a node and an $\operatorname{arc}(i, j)$ with capacity of one corresponds to a vehicle that can execute both jobs ( $i, j$ ) in sequence, satisfying certain time-window restrictions. The minimum number of vehicles equals the minimum number of directed paths such that each node in the network is included in exactly one path. They also develop a polynomial time minimum-flow algorithm to solve the problem.

\subsection{Multi-load capacity vehicles}

The use of multi-load capacity vehicles can reduce the number of vehicles needed or increase the throughput of the system. A multi-load vehicle can pick up additional loads while transporting a previously assigned task. The use of multi-load vehicles can therefore reduce the amount of vehicles' empty trip time and also the total distance traveled is likely to reduce. Bilge and Tanchoco (1997) demonstrate the effectiveness of using multi-load vehicles compared to unit-load vehicles. After experimenting using simulation, they conclude that using multi-load AGVs increases the system throughput, especially in case of high transport demand. Using simulation, Van der Meer and De Koster (1999) also show that multi-load 
vehicles help to increase the system performance, especially, in the case where multiple loads can be picked up at one location. A disadvantage is that a more complex scheduling system is required.

Using estimation models mentioned above, the estimated number of vehicles may considerably differ from the real vehicle requirements due to some impractical assumptions in the analytical models. Moreover, the number of vehicles is strongly affected by the dispatching rules used (Egbelu, 1987), traffic management, congestion and other factors. Therefore, the estimated number should be re-evaluated using a simulation model for specific operational conditions.

\section{Managing automated guided vehicle systems}

\subsection{Vehicle scheduling system}

The vehicle scheduling system decides when, where and how a vehicle should act to perform tasks, including the route it should take. If all tasks are known prior to the planning period, the scheduling problem can be solved offline. However in practice, exact information about jobs (tasks) is usually known at a very late instant. This makes offline scheduling hardly possible. Therefore, online scheduling or dispatching systems are needed to control vehicles.

\subsubsection{Offline scheduling system}

In the offline case, all transportation requests are known in advance. The complete vehicle routes can be optimized and constructed before vehicles carry them out. A small change in job arrival time, a change in driving time (congestion), or failure of a vehicle may impact or even destroy the whole schedule. The scheduling problem in an AGV system is similar to the pick-up and delivery problem with time windows (PDPTW), which often has travel time minimization or minimizing the number of vehicles as objectives. However, the vehicle scheduling problem in AGV systems has some characteristics differing from the PDPTW applied in a truck scheduling environment, such as higher traffic density, higher routing variation, shorter planning horizon due to stochastic load arrivals, vehicle interference and battery charging problems.

The PDPTW problem is known to be NP-hard, so it is unlikely that we can find an algorithm to solve this type of problem in polynomial time. Due to this reason heuristics are the most appropriate approach to cope with this type of problem. Dumas et al. (1991) develop an exact 
algorithm, which uses a column generation scheme with a constrained shortest-path as a subproblem, to solve the PDPTW. The objective is to minimize the sum of the total travel cost. Only homogeneous vehicles (that is of a single type) are considered. Savelsbergh and Sol (1995) provide a survey on the general pick-up and delivery problem (GPDP).

In manufacturing environments, the vehicle schedule is affected by the machine schedule. Hence, in this type of environment, the scheduling system needs to have the capability to schedule both systems at the same time. Bilge and Ulusoy (1995) formulate the integrated scheduling problem of machines and the AGV system as a MIP formulation. Since the problem is very difficult to solve by an exact method, they propose a heuristic for the solution. The heuristic solves two scheduling problems (machines and AGVs) iteratively until a sufficiently good result is obtained. Ulusoy et al. (1997) improve the solution quality by using a genetic algorithm to solve the integrated scheduling problem.

\subsubsection{Online scheduling system}

In practice, environments are usually stochastic (job arrivals, the travel time, loading and unloading times fluctuate, vehicles can breakdown), so the schedule has to be adapted dynamically in time. Savelsbergh and Sol (1995) discuss several solution approaches for the dynamic PDPTW. The schedule of vehicles should be updated when new transportation request information arrives. An approach is to schedule vehicles using a rolling horizon in which vehicle routes are updated after a predetermined time period (time horizon). Savelsbergh and Sol (1998) develop a set-partitioning model for a real PDPTW a Dutch parcel carrier. They develop a branch-and-price algorithm to solve this problem dynamically. Yang et al. (2002) propose several policies to schedule vehicles dynamically. Their model (the truck-load pick-up-and-delivery problem with time windows) is described below:

$$
\operatorname{Min} \sum_{k=1}^{K} \sum_{i=1}^{N} c_{0 i}^{k} x_{k, K+i}+\sum_{i=1}^{N}\left(\alpha \omega_{i} x_{K+i, K+i}+\sum_{j=1, j \neq i}^{N} c_{i j} x_{K+i, K+j}\right)+p \sum_{i=1}^{N} t_{i}
$$

subject to:

$$
\begin{aligned}
& \begin{array}{llll}
\sum_{v=1}^{K+N} x_{u v}=1 & \forall u=1, . ., K+N \text { (1) } & x_{u v}=0,1 & \forall u, v=1, . ., K+N \\
\sum_{v=1}^{K+N} x_{v u}=1 & \forall u=1, . ., K+N(2) & -\sum_{k=1}^{K}\left(d_{0 i}^{k}+\theta_{k}\right) x_{k, K+i}+t_{i} \geq 0 & \forall i=1, . ., N
\end{array} \\
& \left(\omega_{i}+d_{i j}\right) x_{K+i, K+i}-T x_{K+i, K+j}-t_{i}+t_{j} \geq-T+\omega_{i}+d_{i j} \quad \forall i, j=1, . ., N \\
& \tau_{i}^{-} \leq t_{i} \leq \tau_{i}^{+} \\
& \forall i=1, . ., N
\end{aligned}
$$


where $K$ - number of trucks (truck $k$ is first available at time $\theta_{k}$ and at location $o_{k}$ ); $N$ - number of known demands; demand $i$ required to move from a origin $a_{i}$ to a destination $b_{i}$ between time windows $\left[\tau_{i}^{-}, \tau_{i}^{+}\right], t_{i}{ }^{-}$ service time at node $i$; $C(a, b)$ - cost of empty travel between two points $a$ and $b=1 \times D(a, b) ; D(a, b)$ - distance between two points $a$ and $b ; \omega_{i}\left(=D\left(a_{i}, b_{i}\right)\right)$-required loaded distance to serve demand $i$; $p$ - penalty coefficient; $\alpha \omega_{i^{-}}$the lost revenue for rejecting demand $i$ ( $\alpha$ - a positive constant); $d_{0 i}^{k}=D\left(o_{k}, a_{i}\right), c_{0 i}^{k}=C\left(o_{k}, a_{i}\right), k=1 . . K, i=1 . . N$ - distance and cost matrices for a truck $k$ and a demand $i ; d_{i j}=D\left(b_{i}, a_{j}\right), c_{i j}=C\left(b_{i}, a_{j}\right), j=1 . . N, i=1 . . N$ - distance and cost matrices for other demands; $T$ - a large number. The binary variable $x_{k, K+i}$ is to indicate whether truck $k$ first serves demand $i$; $X_{K+i, K+j}$ is to indicate whether there is a truck that serves demand $i$ and demand $j$ consecutively; $x_{k, k}=1$ means that truck $k$ serves no demand; and $X_{K+i, K+i}=1$ means that demand $i$ is rejected.

The objective is to minimize the total cost of processing all demands, which is a combination of the cost of empty travel distance, of penalty for delay, and of lost revenue due to loads rejected. Due to the constraints (1-3), the solution will constitute a feasible assignment. The constraints (4-5) disallow any cycle without a truck. The constraint (6) is a time-window constraint. This model can be seen as a model to schedule vehicles in an AGV system using unit-load vehicles. For a real-time situation, this problem is solved every time a new request for service is received (they call this the “OPTUN” policy) and as a result, new assignments are made. This problem was solved using CPLEX. Since this problem is hard to solve for big instances, the input for the problem is restricted to a few known jobs. Other policies in their paper assign a new load to a vehicle based on a specific criterion such as total cost. They show for a test problem, that OPTUN outperforms simple policies. The OPTUN policy has limited applications since CPLEX cannot solve big instances. OPTUN also uses some probabilistic information of future jobs which certainly improve solution quality.

Meersmans (2002) proposes a heuristic based on a beam-search algorithm to dynamically schedule AGVs at a container terminal. The quality of the schedule depends on the length of the planning horizon (the scheduling problem takes into account only known jobs during that time period) and the rescheduling frequency (the frequency at which the schedule is regenerated). He observes that the longer the planning horizon and the higher the frequency of rescheduling, the better the performance. Other vehicles assignment strategies have been proposed by Fleischmann et al. (2003) and Powell et al. (2000).

\subsubsection{Vehicle dispatching system}

Online dispatching rules are simple and can be easily adapted for automated guided vehicle management systems. The common objectives are minimizing load waiting time, maximizing system throughput, minimizing queue length, or guaranteeing a certain service level at 
stations. There are two main types of online dispatching systems: decentralized and centralized systems.

\section{口 Decentralized control system}

Decentralized control systems dispatch vehicles based on local information only. There is no system to coordinate between AGVs and the central control system. Traditionally, vehicle systems have been implemented and analyzed assuming that every vehicle is allowed to visit any $\mathrm{P} / \mathrm{D}$ location in the system. One of the simplest implementations is one in which vehicles circulate in a unidirectional single-loop.

Bartholdi III and Platzman (1989) study a decentralized heuristic to control AGVs in a simple loop. In their research, an AGV, which can carry up to three loads, travels in a simple unidirectional loop and transports loads according to the FEFS rule. With the FEFS rule, the AGV circulates a loop continuously. Whenever the vehicle has space available, it picks up the first load it encounters, which will then be delivered whenever the destination is reached. Sinriech and Tanchoco (1992b) provide another study that investigates the performance of single-loop systems.

The smart vehicles mentioned in the introduction are modern examples of decentralized control vehicles. Berman and Edan (2002) propose a hierarchical, fuzzy behavior-based methodology to control a AGV system. Central knowledge about the system's state is not available. Agents, representing the smart AGVs, collect the workstations' statuses directly and dynamically decide their next task. Lindeijer (2003) also uses agent-technology to determine the best, conflict-free route an AGV can take (see section 4.4). The agenttechnology becomes more and more important to control AGVs in intelligent manufacturing systems (Shen and Norrie, 1999).

\section{a Centralized control system}

In centralized control systems, a central controller keeps track of all movements regarding internal transport. All information related to vehicles such as pick-up and delivery locations, load-release times, vehicle positions and status, are stored in the controller's database. The controller assigns loads to vehicles (or vice versa) according to specified rules. The centralized controller continuously communicates with vehicles to guide them. Depending on the ways in which transportation requests are assigned, the dispatching rules can be divided into two categories (Egbelu and Tanchoco, 1984): workstation-initiated (jobs at a workstation 
have the priority to claim vehicles) and vehicle-initiated dispatching rules (vehicles have the priority to claim jobs). In this paper, we classify vehicle-dispatching rules as single-attribute, multi-attribute, hierarchical, look-ahead and pre-emption dispatching rules.

\section{$\underline{\text { Single-attribute dispatching rules }}$}

Single-attribute dispatching rules dispatch vehicles based on one parameter/criterion only. Parameters can be travel distance (distance-based), queue length (workload-based), load waiting time (time-based), or other criteria such as a rule based on vehicle availability (by Talbot, 2003).

Distance-based dispatching rules dispatch vehicles based on travel distances or travel times. This category includes rules such as shortest-travel-time(distance)-first (STT(D)F) or nearestwork-station-first (NWF), and nearest-vehicle-first (NVF). According to the shortest-traveltime-first (STTF) rule (Egbelu and Tanchoco, 1984), a vehicle is sent to the closest load to be transported. The closeness of a load can be defined in terms of travel time or distance. This rule leads to little empty travel time of vehicles, but is sensitive to the layout of load locations in the facility (Egbelu and Tanchoco, 1984; De Koster et al., 2004).

Workload-based dispatching rules take queue sizes (or workloads of workstations) into account. In Egbelu and Tanchoco (1984) and Sabuncuoglu (1998), several queue-size rules are introduced, such as the maximum-outgoing-queue-size (MOQS) rule and the minimumremaining-outgoing-queue-space (MROQS) rule. The MOQS rule dispatches a vehicle to the workstation with the largest number of loads waiting to be picked up in its outgoing queue. MROQS dispatches vehicles to the workstation with the minimum remaining space in its outgoing queue. The aim of this rule is to reduce the possibility of queue overflowing or workstation blocking. In addition, several rules based on vehicle utilization (such as select the least utilized vehicle) are proposed by Egbelu and Tanchoco (1984) and Mahadevan and Narendran (1994).

Time-based dispatching rules dispatch vehicles based on jobs' waiting time. These rules include the first-come-first-served (FCFS) rule and two modified first-come-first-served rules (MFCFS, MODFCFS) (see Egbelu and Tanchoco, 1984; Srinivasan et al., 1994). Under the MFCFS rule, a station places only one move request at a time, so it can have at most one "active" move request. The MODFCFS rule differs from the MFCFS rule as it attempts to reduce unnecessary empty travel time by allowing the vehicle to override the FCFS rule whenever it finds an unassigned move request at the destination point. Yamashita (2001) provides an analytical analysis of AGV systems using FCFS dispatching policies. 
De Koster et al. (2004) have carried out extensive simulation experiments with several commonly used dispatching rules such as NVF, for three real cases. They experimented with different operating conditions for AGV systems and point out that for environments where the queue space is critical, the distance-based dispatching rules (NWF, NVF) outperform other rules. However, when it is not the case, the time-based (MODFCFS) or workload-based dispatching rules might perform better.

\section{Multi-attribute dispatching rules}

Multi-attribute dispatching rules dispatch vehicles using more than one parameter (Klein and Kim, 1996; Hwang and Kim, 1998; Jeong and Randhawa, 2001). In general they outperform single-attribute dispatching rules. Klein and Kim (1996) propose several multi-attribute dispatching rules that are based on the multi-criteria decision making approach. Hwang and Kim (1998) propose a multi-attribute dispatching rule based on the bidding concept. Jeong and Randhawa (2001) propose multi-attribute dispatching rules that use three parameters: vehicle empty travel distance, remaining spaces in input buffers and remaining spaces in outgoing buffers to decide which load should be transported by a vehicle. They use an additive waiting model to compute weights for member parameters. A neural network was used to dynamically adjust the parameters' weights reflecting changes in the system. According to their results, a simple multi-attribute dispatching rule with a good set of weights might perform very well and is better in many cases than a multi-attribute dispatching rule with dynamically adjusted weights. Jeong and Randhawa (2001) have done a quite extensive simulation study, however only one layout was used in their experiments.

\section{$\underline{\text { Hierarchical dispatching rules }}$}

This type of dispatching rules is typical for manufacturing systems where the added value of a part during the manufacturing process is taken into account when the dispatching decision has to be made. Sabuncuoglu and Hommertzheim (1992) use a dynamic dispatching algorithm for scheduling machines and AGVs in a flexible manufacturing system (FMS). In their algorithm, different decision criteria are applied sequentially to identify the most appropriate part and the machine to be served. They identify four hierarchical logic levels: push logic, buffer logic, pull logic and push-pull logic. At each logic level, some priority rules are applied to select the part and the machine. Their algorithm performs quite well compared with simpler dispatching rules. Similar approaches have been proposed for

scheduling AGVs in FMS by Yim and Linn (1993), Taghaboni (1997) and Tan and Tang (2001). Kim et al. (1999) introduce a hierarchical rule based on workload balancing. At the 
first level, the jobs are prioritized and at the second level a vehicle is assigned to the job with the highest priority. A complex priority index based on workload balancing among machines (dominant factor) and the urgency of jobs is defined.

Dispatching rules using a look-ahead period, or vehicle reassignment (pre-emption)

Bozer and Yen (1996) introduce two dispatching rules that consider reassignment of moving vehicles. These are modified shortest-travel-distance-first (MOD STTF) and bidding-based device dispatching $\left(\mathrm{B}^{2} \mathrm{D}^{2}\right)$. The MOD STTF rule is similar to the STTF rule in the sense that it assigns empty vehicles to move requests based on the proximity of the vehicle and the load location, and each vehicle has only one request at a time. The difference is that an empty vehicle may be reassigned to another move request or an empty vehicle may "release" another empty vehicle. If a vehicle travels "uncommitted" to its assigned destination, it may be reassigned to a new arrival request according to some specific conditions (Bozer and Yen, 1996). To some extent, the $B^{2} D^{2}$ rule is similar to the MOD STTF rule, but it is much more complicated. Using a quite extensive simulation study (four layouts and a large set of experimental conditions) Bozer and Yen (1996) show that MOD STTF and $\mathrm{B}^{2} \mathrm{D}^{2}$ perform much better than STTF.

Look-ahead dispatching rules use some advance information about loads to be available shortly to dispatch vehicles (Mantel and Landeweerd, 1995). De Koster et al. (2004) improve the AGV system performance using dispatching rules with prior information on the availability of loads. They experiment with several widely used dispatching rules (NWF, MODFCFS etc.) with and without pre-arrival information. According to their simulation experiments, a very short look-ahead period has significant positive effects on the system performance.

Table 4 A guideline for selecting an appropriate vehicle scheduling system

\begin{tabular}{|c|c|c|c|c|c|c|c|c|c|c|}
\hline \multicolumn{2}{|c|}{ Criteria } \\
\hline \multicolumn{2}{|c|}{ Guide-path system } & $\begin{array}{c}\text { Ability of SFC } \\
\text { system to deal with } \\
\text { complicated } \\
\text { controllers }\end{array}$ & \multicolumn{5}{|c|}{$\begin{array}{c}\text { Degree of } \\
\text { stochasticity } \\
\text { of jobs' arrivals }\end{array}$} & \multicolumn{3}{c|}{ Job density } \\
\hline $\begin{array}{c}\text { Single } \\
\text { loop }\end{array}$ & $\begin{array}{c}\text { Tan- } \\
\text { dem }\end{array}$ & $\begin{array}{c}\text { Conven- } \\
\text { tional }\end{array}$ & Yes & No & No & Low & Medium & High & Low & High \\
\hline D & D & S/ D & S/ D & D & S(off) & S(on) & S(on)/ D & S(on)/D & S (r)/ D & S/D(r) \\
\hline
\end{tabular}

*SFC: Shop floor control; D: dispatching; S: scheduling; on: online; off: offline; r: recommended.

Table 4 presents a guideline for designers to choose a suitable vehicle scheduling system for implementation. Vehicles in simple guide-path systems (single-loop, tandem) can be 
dispatched using simple dispatching rules without reducing the system performance. In practice, the available SFC system may not have the capability to deal with a complicated controller. In this case, a scheduling system that requires more information and advanced monitoring systems may not be applicable. In highly stochastic environments, it is impossible to schedule vehicles over a long horizon, so dispatching rules might be a better option in this case. In case of a high job density, vehicles are busy most of the time so implementing a complicated scheduling system will not be very helpful.

Because of their simplicity, vehicle dispatching rules are easy to implement. However, as indicated by Yang et al. (1999) and Meersmans (2002), dynamic vehicle scheduling is often more efficient. Meersmans (2002) also indicates that dynamic vehicle scheduling has the capability of taking other factors such as co-ordination between different transportation means in facilities into account.

Another issue, which has to be considered in the scheduling, is vehicle parking. Most scheduling problems suppose that vehicles can stay at the load's pick-up/drop-off locations, however this is not true in some AGV systems. Hence, vehicle parking problem should also be included in the vehicle scheduling problem. This is considered in the next section.

\subsection{Vehicle positioning strategy}

Vehicle idleness is unavoidable in automated guided vehicle systems. Rather than forcing vehicles to return to the vehicle depot, it is better to park vehicles at locations (vehicle home locations or dwell points) that are closer to load-release locations than the vehicle depot. Two main strategies for idle-vehicle positioning (parking) are static and dynamic strategies.

\subsubsection{Static vehicle positioning strategy}

Vehicle parking locations should be selected to minimize the vehicle response time to new movement requests or to evenly distribute idle vehicles over the network. Several positioning strategies are proposed in literature (Egbelu, 1993; Van der Meer, 2000). Four major approaches are:

Central-zone positioning rule: a certain parking area in the vehicle network has been designated for buffering idle vehicles. This area can be close to stations with a high probability of a load transport request, or at battery- recharge or fuel stations.

Circulatory-loop positioning rule: one or more cruising loops are defined for idle vehicles. When a vehicle becomes idle, it travels one of the loops until a transport order is received. 
Drop-off point positioning rule: a vehicle remains at the point of the last delivery job until it is reassigned.

Distributed-positioning rule: a distributed-positioning rule employs multiple dwell points as opposed to a single point, as in the central zone case. When a vehicle becomes idle, it is routed to one of the dwell points.

Most literature that discusses dwell-point strategies for automated guided vehicle systems, involves selecting home locations of vehicles in a single-loop. A common approach in finding home locations of vehicles in a single-loop is translating the loop layout into a circular layout and after this step all following calculations are based on angular positions. Two other approaches use Markov chain theory (Kim and Kim, 1997) and network flow modeling (Hu and Egbelu, 2000). Egbelu (1993) uses the circular layout conversion to search for the best home locations for idle vehicles. He proposes four models and solution methods: for a single vehicle in a unidirectional loop and in a bidirectional loop, and for multiple vehicles in a unidirectional loop and in a bidirectional loop. The objective of the model is to minimize the maximum response time of the idle vehicles. The objective function of the simplest case (single vehicle, unidirectional loop) is stated as:

$$
T=\min \left\{\max _{1 \leq i \leq n}\left\{\frac{R}{V}\left[\left(\alpha_{i}-\beta\right)\left(1-X_{i}\right)+\left(360+\alpha_{i}-\beta\right) X_{i}\right]\right\}\right\}
$$

where $\alpha_{i}$ : angular location of the $i_{\text {th }}$ workstation; $\beta$ : angular location of the vehicle; $R=C / 360^{\circ}$ (C: the total length or perimeter of a guide-path that describes the loop) ; $V$ : the average speed of the vehicle; $X_{i}=1$ if $\alpha_{i} \geq$ $\beta,=0$ otherwise.

According to Egbelu (1993), the optimal home location of a vehicle in the unidirectional loop coincides with the location of a workstation and the optimal home location of a vehicle in the bidirectional loop lies at the midpoint of an arc. Based on these characteristics and the traffic flow of the system, Egbelu proposes several algorithms to find the optimal location for idle vehicles. He also indicates that it is extremely difficult to control multiple vehicles in the bidirectional loop. Kim (1995) proposes a similar approach to minimize the mean response time for a pick up call and a single parking place policy is used. Gademann and Van de Velde (2000) consider the problem of positioning $m$ AGVs in a loop layout with $n$ stations. They provide an overview of time complexities for uni-directional and bidirectional flow systems and show that criteria like maximum response time and average response time can be minimized in polynomial time for any number of vehicles. Lee and Ventura (2001) propose a polynomial-time dynamic-programming algorithm that determines the optimal dwell-points of idle AGVs for both unidirectional and bidirectional loop layouts. The objective is 
minimizing the mean response time. Their algorithm decomposes the set of pick-up stations into subsets so that a single vehicle serves all stations in a subset.

Hu and Egbelu (2000) propose network-flow based models for selecting the optimal parking locations for idle vehicles in a unidirectional network (not necessarily to be a loop). The objectives are minimizing the maximum response time and minimizing the mean response time. A formulation with the non-linear objective function is presented below ( $\mathrm{Hu}$ and Egbelu, 2000):

minimize $\left\{\max _{\forall i, j}\left\{d_{i j} y_{i j}\right\}\right\}$ (minimization of maximum system response time)

$$
\begin{aligned}
& \text { subject to: } \quad \sum_{i=1}^{N} x_{i}=n \quad \text { (1) } \quad \sum_{j=1}^{N} y_{i j} \leq m x_{i} \quad i=1,2, \ldots, N \\
& \sum_{i=1}^{N} y_{i j}=1 \quad j=1,2, \ldots, m(2) \quad x_{i}, y_{i j}=0 \text { or } 1, \forall i, j
\end{aligned}
$$

where $x_{i}:=1$ if node $i$ is selected as a dwell point, $=0$ otherwise; $y_{i j}=1$ if dwell point $i$ serves pick-up station $j,=0$ otherwise; $m$ : the number of pick-up stations; $n$ : the number of vehicles; $N$ : the number of possible dwell points; $d_{i j}$ : a shortest distance matrix of the modified network (obtained from the origin network after applying the network reconfiguration procedure) which is free from convergent nodes.

This formulation is then transformed into a MIP model by replacing the objective function with expressions: ( $\min Z$, s.t. $d_{i j} y_{i j} \leq Z$ ). Two solution procedures, one exact and one heuristic, are proposed to solve the problem. The exact method solves a sequence of set covering problems. After finding the optimal locations for vehicles they present a six-steps procedure to distribute vehicles among dwell points. For minimizing the mean response time, a linear integer program is given. This model formulation is similar to the formulation of the p-median problem and solved using a branch-and bound-method. After finding the optimal dwell points, vehicles are distributed by the same procedure used for the previous case.

\subsubsection{Dynamic vehicle positioning strategy}

When pick up demands at the stations change over time, the home locations of vehicles may need to be changed. In order to adapt to this situation some dynamic procedures for selecting dwell points are proposed. Kim (1995) adapts his static algorithms to cope with the dynamic situation. Kim's algorithm bases on the calculation of the contribution of each segment on the circular layout to the mean angular travel distance. Chang and Egbelu (1996) propose dynamic algorithms to select the home location of a single vehicle in the unidirectional and bidirectional loop. They show that, in a very busy system, the performance is independent of 
the dwell point selection rules. Hu and Egbelu (2000) extend their algorithms to the situation where the pick-up demands change over time in a non-uniform manner. Their method requires an accurate update of load pick-ups remaining at each station at event times. When the ratio of remaining load pick-ups changes, the idle-vehicle positioning problem has to be solved again. Their algorithm applies for minimizing both the maximum response time and the mean response time.

Most studies in this area concentrate on loop layouts only. The research by $\mathrm{Hu}$ and Egbelu (2000) is the only one that takes conventional layouts into account. In practice, companies may define fixed parking locations, because vehicles may or can only park in certain areas. These areas can be defined for safety reasons, to avoid congestion, to allow a change of drivers, to recharge the vehicle's battery, etc. and impose constraints on the idle vehicle positioning problem. Such practical issues are often overlooked or omitted in theoretical models of automated material handling systems.

\subsection{Battery management}

Although battery management is important for vehicle management, the battery management problem is usually omitted in research. Naturally, vehicles have to be charged after a certain operating period, but most research on automated material handling systems assumes that the battery problem has little effect on performance. However, in reality there is a potential impact on performance as vehicles with nearly empty batteries are unavailable for the process, even if swap batteries are used. Battery swapping can only be carried out at specific locations, so vehicles are temporarily unavailable. This means that either additional vehicles are needed or load-waiting times increase. According to McHaney (1995), the batteries' constraints can only be omitted under some circumstances: systems with naturally occurring breaks, or shift changes coinciding with battery swapping or charging, systems with ample amounts of idle time, and systems where charging can be regulated and insured to take place without impacting system operation. McHaney (1995) presents three types of charging schemes: (1) opportunity charging - uses the natural idle time in an AGV's cycle to replenish batteries, (2) automatic charging - an AGV runs until its battery is depleted to a certain level and then the scheduler assigns this AGV for charging, (3) combination system - this is a combination of the previous two. 
Ebben (2001) suggests several heuristic rules for dispatching vehicles, which need to be charged. It is possible to send vehicles to the nearest battery station, farthest reachable battery station on the current route, etc. In addition, we also have to consider the capacity of the battery charging stations (are there sufficient charging positions for vehicles), and the vehicle's next job, so we can add some other rules, such as go to the battery station closest to the vehicle's next job.

The problem of estimating the number of batteries required is also important. The required number of batteries strongly depends on the chosen battery type (Ebben, 2001). Ebben (2001) also shows that the required number of battery changes largely depends on the net capacity of the battery and less on the number of battery stations. He also proposes a cost trade-off analysis to help the designer to choose the battery's type, the number and position of battery stations.

Another issue here is how to select locations for battery-charging stations. They have to be located to minimize battery-charging effects on the system operation. In practice, battery stations often coincide with the vehicle parking locations to save spaces and use the positions for opportunity charging. The vehicle's battery charging scheme should also be considered explicitly when vehicles are scheduled for operations. These issues are not considered in the literature at this stage.

\subsection{Vehicle routing and conflict resolution}

At the operational decision level, the vehicle routing and conflict resolution problem has to be addressed. Scheduling and routing the vehicles in internal transport systems without conflict is very important. A conflict may cause the whole system to collapse or to become blocked. Many causes can lead to a deadlock situation, i.e. when two vehicles arrive at the same intersection from different guide-path segments and cannot proceed without interference. There are several ways to avoid deadlock and conflict in automated guided vehicle systems for example using a better routing algorithm, using single-loop, tandem or SFT configurations; identification of imminent collision through forward sensing and consequently avoiding this through vehicle backtracking and/or rerouting; imposing zone control and extensive route pre-planning. 


\subsubsection{Vehicle routing}

The vehicle scheduling and routing problems are closely related and should be addressed concurrently. In tandem systems, the routing problem is very simple, but in conventional systems, it is more complicated. In the scheduling section (4.1), we supposed that a vehicle could reach its destination without blocking. However, to avoid blocking, the vehicle routing needs to be taken into account as well. Kim and Tanchoco (1991) propose an algorithm based on Dijkstra's shortest-path method to schedule vehicles based on the nodes' time windows. This approach produces a conflict-free schedule. However, as noted by the authors, a small change in the schedule may destroy it completely. Taghaboni and Tanchoco (1995) introduce an incremental route planning and scheduling algorithm. Other approaches are introduced in Rajotia et al. (1998b) and Qiu and Hsu (2001). More details about the vehicle routing issue can be found in Qiu et al. (2002).

\subsubsection{Conflict resolution}

\section{a Balancing the system workload}

When machines in a manufacturing system or the P/D locations in a distribution center have only little space for load buffering, the system might be blocked by buffers overflowing. Possible reasons include insufficient buffering capacity or an inappropriate scheduling (dispatching) system. To cope with the first problem, a central buffer may be introduced to solve the temporary blocking problem. Kim et al. (1999) show that the central buffer has an important influence on the system performance. The second problem can be solved by applying workload-related dispatching rules (Egbelu and Tanchoco, 1984; Mahadevan and Narendran, 1994; Kim et al., 1999).

\section{a Forward sensing}

AGVs can be equipped with sensors which they can use to detect if they are too close to other vehicles (Zeng et al., 1991). A vehicle stops when the distance between it and the vehicle in front of it, is less than a threshold value. This technique however is not effective for systems with many curved guide-paths. Zeng et al. (1991) use Petri nets to detect conflicts in AGV systems. A Petri-net approach seems to be a promising direction to detect and prevent conflicts in AGV systems (Zeng et al., 1991; Hsieh and Kang, 1998).

\section{a Control the traffic at intersections}


Routing vehicles through intersections is a key issue in the conflict resolution. Egbelu and Tanchoco (1986) develop an algorithm to route vehicles through an intersection without conflict. Their model assumes that all nodes have buffers, that the buffers are of infinite capacity and the time required for a vehicle to steer into and out of the buffer is small compared to the overall travel time required. Also in their paper, several types of buffering areas for vehicles in transit are designed. They include "loop”, "siding” and "spur" designs. The buffering areas provide space to avoid the blocking situation at intersections. The main idea in the deadlock resolution at an intersection is to buffer selected vehicles and gradually resolve conflicts. Evers and Koppers (1996) introduce the concept of "semaphore" as an abstraction of a traffic light to control vehicles at intersections. The number of vehicles controlled by a semaphore cannot exceed a specified maximum, which is called the capacity of the semaphore. A semaphore, in their paper, is a non-negative integer variable (S), with the interpretation of free capacity, on which two operations are defined: "Wait" and "Signal". Operation "Wait" is executed when a vehicle arrives at the protected facility, whereas "Signal" is executed when the vehicle leaves the protected facility.

\section{¿ Zone planning}

Zone planning is an important method to avoid deadlock. There are two types of zoning systems: static zoning and dynamic zoning. In case of a static zoning, the guide-paths are divided into several zones. When a vehicle arrives at a zone, the controller checks for the presence of another vehicle in this zone. If a vehicle is already traveling in this zone, then the vehicle intended to enter that zone has to wait until the other has passed. In case of a dynamic zoning strategy, zones are not fixed; they can be changed according to the traffic flow in the system. Ho (2000) presents a dynamic-zone strategy for vehicle-collision prevention. His method relies on two procedures: the Zone Adjustment Procedure and the Zone Assistance Procedure. With the Zone Adjustment Procedure, the area of each zone changes according to the current production demand. The Zone Assistance Procedure allows vehicles to help each other so that the workload of every vehicle is balanced over time. Reveliotis (2000) proposes a zone control strategy that determines vehicle routes incrementally, one zone at a time. Routing decisions are the results of a sequence of safety and performance considerations, with the former being primarily based on structural/ logical rather than timing aspects of the system behavior.

In general, the conflict resolution task is much simpler in systems using smart AGVs, since smart AGVs can handle most parts of collision avoidance tasks. 


\section{A decision framework for AGV systems' design and implementation}

Our literature review sketches a hierarchical structure for AGV systems' design and implementation decisions. Figure 3 summarizes these decisions. Decision at a higher level set the boundary conditions for decisions at a lower hierarchical level which are taken later.

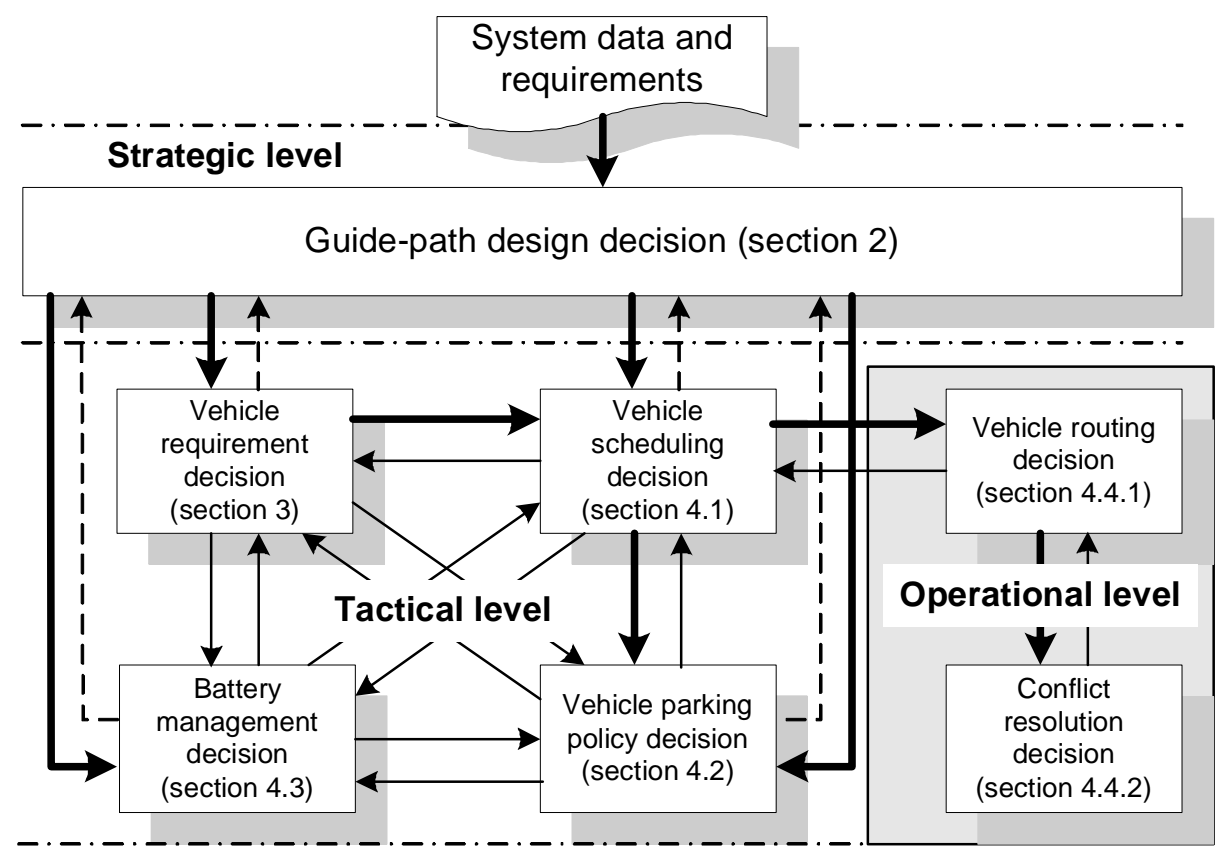

Arrows indicate interactions between decisions: thick, thin, and dash arrows mean strong, less strong, and possible less strong influences.

Figure 3 The decision framework for design and implementation of AGV systems

The design process requires input data such as facility layout and performance requirements in order to start. The first step at strategic level is designing the guide-path system. After this step, we consider issues at tactical level including estimating the number of required vehicles, scheduling vehicles, deciding the parking policy, and designing the battery management scheme. Decisions at tactical level influence each other and should be considered simultaneously. These decisions may also influence the guide-path design decision (represented by dash arrows in Figure 3), particularly in case free-ranging or smart AGVs are used. At operational level, we need to design a system to guide vehicles to their destination without conflict.

\section{Conclusions and further research}

In this paper, we have discussed several key issues in AGV system design and implementation. These issues include guide-path design, estimating the required number of 
vehicles, vehicle scheduling (dispatching), idle vehicle positioning, battery management, vehicle routing, and conflict resolution. For every decision problem we have reviewed important literature and have classified decision problems.

The first important issue when designing AGV systems is guide-path design. In general, we can divide guide-path systems into three categories: conventional, single-loop and tandem systems. Each of them has its own advantages and disadvantages (Table 3) and is suitable for specific applications.

Next important issue is estimating the required number of vehicles. Main vehicle estimating models include travel time (Maxwell and Muckstadt, 1982), queueing (Tanchoco et al., 1987), regression (Arifin and Egbelu, 2000) and network (Vis et al., 2001) models. Obtaining a good estimation of the vehicle empty travel under some good dispatching rules is the key problem here.

Another issue is how to manage an AGV system efficiently. This issue includes several subissues: vehicle scheduling, idle vehicle positioning, battery-charging, vehicle routing and conflict resolution. In practice, online scheduling and dispatching systems are much more popular than offline scheduling due to the stochastic nature of AGV systems. Generally, online scheduling systems perform better than simple dispatching systems (Yang et al., 1999), however they are much more complex. The scheduling system also decides which route a vehicle should take to reach its destination without collisions. In cases where vehicle blocking and interference problems are likely, we need a good conflict resolution system. In addition, to better serve future jobs, the controller should guide idle vehicles to strategic parking locations. Vehicles are usually located in such a way that the mean or maximum response time of new requests is minimized. Most authors neglect AGV battery management problems. In the case of long travel distances, the battery charging becomes an issue and needs to be considered as well.

\section{Suggestions for further research}

Several future research subjects have been proposed in the previous sections. Here we repeat the most promising future research directions.

- In section 2, we have seen three main types of guide-path systems. It is important to know when and where we should choose a specific guide-path system. In addition, the bidirectional guide-path system has not received much attention, in spite of the fact that it can improve the system performance (Egbelu and Tanchoco, 1986). 
- For estimating the required number of vehicles, obtaining a better estimation of the vehicle empty travel and time lost caused by vehicle interference under some good dispatching rules is still an important issue.

- Bilge and Tanchoco (1997) show that using multi-load vehicles improves the system throughput. In literature, there are only few studies considering multi-load AGV systems (Sinriech and Palni, 1998; Sinriech and Kotlarski, 2002; Van der Meer and De Koster, 1999). Multi-load vehicles are expensive, but can lead to a substantial throughput increase, depending on the quality of the scheduling system. This leaves ample room for future research.

- Smart AGVs become more and more popular in practice. They change the importance of some design and implementation issues in AGV systems. For example, obtaining an optimal guide-path system is less crucial, but the flexibility of layout and system adaptation for future demands might be more important. In addition, agent-like smart AGVs use decentralized control in a more efficient way than just applying FEFS dispatching. Still, there are very few studies related to designing and dispatching smart AGV systems, which clearly gives an important research direction.

- Intelligent manufacturing systems (IMS) using intelligent machines and smart AGVs become increasingly important in practice. In such systems, machines and AGVs can act as intelligent agents. These agents have advanced capabilities such as self-learning lead to adaptiveness and responsiveness capabilities of IMS. Holonic manufacturing system (HMS) is an example of such IMS (Shen and Norrie, 1999). However, more research is still needed to improve performance of IMS and smart AGV agents.

We have pointed out several fruitful issues for future research. These issues certainly have an important impact on improving the AGV system's performance. Besides these issues, researchers should pay attention to other issues such as battery management, positioning idle vehicles and conflict resolution as well.

\section{References}

Al-Sultan, K.S. and Bozer, Y.A. 1998. Network configuration and machine layout in fixed-path material handling systems, Annals of Operations Research 76 21-54.

Arifin, R. and Egbelu, P.J. 2000. Determination of vehicle requirements in automated guided vehicle systems: a statistical approach, Production Planning \& Control 11 258-270.

Asef-Vaziri, A., Laporte, G. and Sriskandarajah, C. 2000. The block layout shortest loop design problem, IIE Transactions 32 727-734. 
Bartholdi III, J.J. and Platzman, L.K. 1989. Decentralized control of automated guided vehicles on a simple loop, IIE Transactions 21 (1) 76-81.

Beamon, B.M. 1998. Performance, reliability, and performability of material handling systems, International Journal of Production Research 36 (2) 377-393.

Berman, S. and Edan, Y. 2002. Decentralized autonomous AGV system for material handling, International Journal of Production Research 40 (15) 3995-4006.

Bilge, Ü. and Tanchoco, J.M.A. 1997. AGV systems with multi-load carriers: Basic issues and potential benefits, Journal of Manufacturing Systems 16 (3) 159-174.

Bilge, Ü. and Ulusoy, G. 1995. A time window approach to simultaneous scheduling of machines and material handling system in an FMS, Operations Research 43 (6) 1058-1070.

Bozer, Y.A. and Srinivasan, M.M. 1991. Tandem configurations for automated guided vehicle systems and the analysis of single vehicle loops, IIE Transactions 23 (1) 72-82.

Bozer, Y.A. and Srinivasan, M.M. 1992. Tandem AGV systems: a partitioning algorithm and performance comparison with conventional AGV systems, European Journal of Operational Research 63 173-191.

Bozer, Y.A. and Yen, C. 1996. Intelligent dispatching rules for trip-based material handling systems, Journal of Manufacturing Systems 15 (4) 226-239.

Chang, S. and Egbelu, P.J. 1996. Dynamic relative positioning of AGVs in a loop layout to minimize mean system response time, International Journal of Production Research 34 (6) 1655-1673.

Chen, M.-T., McGinnis, L. and Zhou, C. 1999. Design and operation of single-loop dual-rail inter-bay material handling system, International Journal of Production Research 37 (10) 2217-2237.

Co, C.G. and Tanchoco, J.M.A. 1991. A review of research on AGVS vehicle management, Engineering Cost and Production Economics 21 35-42.

De Koster, R., Le Anh, T. and Van der Meer, J.R. 2004 (to appear). Testing and classifying vehicle dispatching rules in three real-world settings, Journal of Operations Management.

Dumas, Y., Desrosiers, J. and Soumis, F. 1991. The pickup and delivery problem with time windows, European Journal of Operational Research 54 7-22.

Ebben, M., 2001. Logistic control in automated transportation networks, Dissertation, University of Twente.

Egbelu, P.J. 1987. The Use of non-simulation approaches in estimating vehicle requirements in an automated guided vehicle based transport system, Material Flow 4 17-32.

Egbelu, P.J. 1993. Positioning of automated guided vehicles in a loop layout to improve response time, European Journal of Operational Research 71 (1) 32-44.

Egbelu, P.J. and Tanchoco, J.M.A. 1984. Characterization of automated guided vehicle dispatching rules, International Journal of Production Research 22 (3) 359-374.

Egbelu, P.J. and Tanchoco, J.M.A. 1986. Potentials for bi-directional guide-path for automated guided vehicle based systems, International Journal of Production Research 24 (5) 1075-1097.

Evers, J.J.M. and Koppers, S.A.J. 1996. Automatic guided vehicle traffic control at a container terminal, Transportation Research A 30 (1) 21-34.

Fleischmann, B., Gnutzmann, S. and Sandvoß, E. 2003 (to appear). Dynamic vehicle routing based on on-line traffic information, Tansportation Science.

Gademann, N. and Van de Velde, S. 2000. Positioning automated guided vehicles in a loop layout, European Journal of Operational Research 127 565-573.

Gaskins, R.J. and Tanchoco, J.M.A. 1987. Flow path design for automated guided vehicle systems, International Journal of Production Research 25 (5) 667-676.

Gaskins, R.J., Tanchoco, J.M.A. and Taghaboni, F. 1989. Virtual flow paths for free-ranging automated guided vehicle systems, International Journal of Production Research 27 (1) 91-100.

Goetz, W.G. and Egbelu, P.J. 1990. Guide path design and location of load pick-up/drop-off points for an 
automated guide vehicle system, International Journal of Production Research 28 (5) 927-941.

Ho, Y.-C. 2000. A dynamic-zone strategy for vehicle-collision prevention and load balancing in an AGV system with a single-loop guide path, Computers in Industry 42 159-176.

Hsieh, S. and Kang, M.-Y. 1998. Developing AGVS petri net control models from flowpath nets, Journal of Manufacturing Systems 17 (4) 237-250.

Hu, C.-H. and Egbelu, P.J. 2000. A framework for the selection of idle vehicle home locations in an automated guided vehicle system, International Journal of Production Research 38 (3) 543-562.

Huang, C. 1997. Design of material transportation system for tandem automated guided vehicle systems, International Journal of Production Research 35 (4) 943-953.

Hwang, H. and Kim, S.H. 1998. Development of dispatching rules for automated guided vehicle systems, Journal of Manufacturing Systems 17 (2) 137-143.

Jeong, B.H. and Randhawa, S.U. 2001. A multi-attribute dispatching rule for automated guide vehicle systems, International Journal of Production Research 39 (13) 2817-2832.

Johnson, M.E. and Brandeau, M.L. 1993. An analytic model for design of a multivehicle automated guided vehicle system, Management Science 39 (12) 1477-1489.

Johnson, M.E. and Brandeau, M.L. 1994. An analytic model for design and analysis of single-vehicle asynchronous material handling systems, Transportation Science 28 (4) 337-353.

Jünemann, R. and Schmidt, T. 2000. Materialflußsysteme. Springer.

Kaspi, M., Kesselman, U. and Tanchoco, J.M.A. 2002. Optimal solution for the flow path design problem of a blanced unidirectional AGV system, International Journal of Production Research 40 (2) 389-401.

Kaspi, M. and Tanchoco, J.M.A. 1990. Optimal flow path design of unidirectional AGV systems, International Journal of Production Research 28 (6) 1023-1030.

Kim, C.W. and Tanchoco, J.M.A. 1993. Operational control of a bidirectional automated guided vehicle system, International Journal of Production Research 31 (9) 2123-2138.

Kim, C.W., Tanchoco, J.M.A. and Koo, P.H. 1999. AGV dispatching based on workload balancing, International Journal of Production Research 37 (17) 4053-4066.

Kim, C.W. and Tanchoco, J.M.A. 1991. Conflict-free shortest-time bidirectional AGV routeing, International Journal of Production Research 29 (12) 2377-2391.

Kim, K.H. 1995. Positioning of automated guided vehicles in a loop layout to minimize the mean vehicle response time, International Journal of Production Economics 39 (3) 201-214.

Kim, K.H. and Kim, J.Y. 1997. Estimating mean response time and positioning idle vehicles of automated guided vehicle systems in loop layout, Computers and Industrial Engineering 33 (3-4) 669-672.

Kim, K.H. and Tanchoco, J.M.A. 1993. Economical design of material flow paths, International Journal of Production Research 31 (6) 1387-1407.

King, R.E. and Wilson, C. 1991. A review of automated guided-vehicle sytems design and scheduling, Production Planning \& Control 2 (1) 44-51.

Klein, C.M. and Kim, J. 1996. AGV dispatching, International Journal of Production Research 34 (1) 95-110.

Lee, C. and Ventura, J.A. 2001. Optimal dwell point location of automated guided vehicles to minimize mean response time in a loop layout, International Journal of Production Research 39 (17) 4013-4031.

Lim, J.K., Lim, J.M., Yoshimoto, K., Kim, K.H. and Takahashi, T. 2002. A construction algorithm for designing guide paths of automated guided vehicle systems, International Journal of Production Research 40 (15) 39813994.

Lindeijer, D.G., 2003. Controlling automated traffic agents, Dissertation, Technical University of Delft.

Mahadevan, B. and Narendran, T.T. 1993. Estimation of number of AGVs for an FMS: an analytical model, International Journal of Production Research 31 (7) 1655-1670. 
Mahadevan, B. and Narendran, T.T. 1994. A hybrid modelling approach to the design of an AGV-based material handling system, International Journal of Production Research 32 (9) 2015-2030.

Mantel, R.J. and Landeweerd, H.R.A. 1995. Design and operational control of an AGV system, International Journal of Production Economics 41 (1-3) 257-266.

Maxwell, W.L. and Muckstadt, J.A. 1982. Design of automated guided vehicle systems, IIE Transactions 14 (2) 114-124.

McHaney, R. 1995. Modelling battery constraints in discrete event automated guided vehicles simulations, International Journal of Production Research 33 3023-3040.

Meersmans, P., 2002. Optimization of container handling systems, Dissertation, Erasmus University Rotterdam.

Powell, W.B., Towns, T.T. and Marar, A. 2000. On the value of optimal solutions for dynamic routing and scheduling in the presence of user noncompliance, Transportation Science 34 (1) 67-85.

Qiu, L. and Hsu, W. 2001. A bi-directional path layout for conflict-free routing of AGVs, International Journal of Production Research 39 (10) 2177-2195.

Qiu, L., Hsu, W., Huang, S. and Wang, H. 2002. Scheduling and routing algorithms for AGVs: a survey, International Journal of Production Research 40 (3) 745-760.

Rajotia, S., Shanker, K. and Batra, J.L. 1998b. A semi-dynamic time window constrained routeing strategy in an AGV system, International Journal of Production Research 36 (1) 35-50.

Reveliotis, S.A. 2000. Conflict resolution in AGV systems, IIE Transactions 32 647-659.

Ross, E.A., Mahmoodi, F. and Mosier, C.T. 1996. Tandem configuration automated guided vehicle systems: A comparative study, Decision Sciences 27 (1) 81-102.

Sabuncuoglu, I. 1998. A study of scheduling rules of flexible manufacturing systems: a simulation approach, International Journal of Production Research 36 (2) 527-546.

Sabuncuoglu, I. and Hommertzheim, D.L. 1992. Dynamic dispatching algorithm for scheduling machines and automated guided vehicles in a flexible manufacturing system, International Journal of Production Research 30 (5) 1059-1079.

Savelsbergh, M.W.P. and Sol, M. 1995. The general pickup and delivery problem, Transportation Science 29 (1) 17-29.

Savelsbergh, M. and Sol, M. 1998. DRIVE: Dynamic routing of independent vehicles, Operations Research 46 (4) 474-490.

Shen, W. and Norrie, D.H. 1999. Agent-based systems for intelligent manufacturing: a state-of-the-art survey, Knowledge and Information Systems, an International Journal 1 (2) 129-156.

Shen, Y.-C. and Kobza, J.E. 1998. A dispatching-rule-based algorithm for automated guided vehicle systems design, Production Planning \& Control 9 (1) 47-59.

Sinriech, D. 1995. Network design models for discrete material flow systems: A literature review, International Journal of Advanced Manufacturing Technology 10 277-291.

Sinriech, D. and Kotlarski, J. A dynamic scheduling algorithm for a multiple-load multiple-carrier system. International Journal of Production Research 40 (5), 1065-1080. 2002.

Sinriech, D. and Palni, L. 1998. Scheduling pickup and deliveries in a multiple-load discrete carrier environtment, IIE Transactions 30 1035-1047.

Sinriech, D. and Tanchoco, J.M.A. 1991. Intersection graph method for AGV flow path design, Intenational Journal of Production Research 29 (9) 1725-1732.

Sinriech, D. and Tanchoco, J.M.A. 1992a. An economic model for determining AGV fleet size, International Journal of Production Research 30 (6) 1255-1268.

Sinriech, D. and Tanchoco, J.M.A. 1992b. Impact of empty vehicle flow on performance of single-loop AGV systems, International Journal of Production Research 30 (10) 2237-2252.

Sinriech, D. and Tanchoco, J.M.A. 1993. Solution methods for the mathematical models of single-loop AGV 
systems, International Journal of Production Research 31 705-725.

Sinriech, D. and Tanchoco, J.M.A. 1995. An introduction to segmented flow approach for discrete material flow systems, International Journal of Production Research 33 (12) 3381-3410.

Sinriech, D. and Tanchoco, J.M.A. 1997. Design procedures and implemetation of the segmented flow topology (SFT) for discrete material flow systems, IIE Transactions 29 323-335.

Srinivasan, M.M., Bozer, Y.A. and Cho, M. 1994. Trip-based material handling systems: Throughput capacity analysis, IIE Transactions 26 (1) 70-89.

Taghaboni, F. 1997. A value-added approach for automated guided vehicle task assignment, Journal of Manufacturing Systems 16 (1) 24-34.

Taghaboni, F. and Tanchoco, J.M.A. 1995. Comparison of dynamic routing techniques for automated guided vehicle systems, International Journal of Production Research 33 2653-2669.

Talbot, L., 2003. Design and performance analysis of multistation automated guided vehicle systems, Dissertation, Universite Catholique de Louvain.

Tan, K.K. and Tang, K.Z. 2001. Vehicle dispatching system based on Taguchi-tuned fuzzy rules, European Journal of Operational Research 128 545-557.

Tanchoco, J.M.A., Egbelu, P.J. and Taghaboni, F. 1987. Determination of the total number of vehicles in an AGV-based material transport system, Material Flow 4 33-51.

Tanchoco, J.M.A. and Sinriech, D. 1992. OSL - optimal single-loop guide paths for AGVS, International Journal of Production Research 30 (3) 665-681.

Tompkins, J.A., White, J.A., Bozer, Y.A. and Tanchoco, J.M.A. 2003. Facilities planning, 3rd edn. John Wiley \& Sons, Inc.

Ulusoy, G., Sivrikaya-Serifoglu§, F. and Bilge, Ü. 1997. A genetic algorithm approach to the simultaneous scheduling of machines and automated guided vehicles, Computers Operations Research 24 (4) 335-351.

Van der Meer, J.R., 2000. Operational control of internal transport system, Dissertation, Erasmus University Rotterdam.

Van der Meer, J.R. and De Koster, R. 1999. Using multiple load vehicles for internal transport with batch arrivals of loads. In: Advances in Distribution Logistics (ed M.Grazia Speranza and P.Stähly (Eds.)). Springer Berlin, pp. 197-214.

Vis, I.F.A., de Koster, R., Roodbergen, K.J. and Peeters, L.W.P. 2001. Determination of the number of automated guided vehicles required at a semi-automated container terminal, Journal of Operational Research society 52 (4) 409-417.

Yamashita, H. 2001. Analysis of dispatching rules of AGV systems with multiple vehicles, IIE Transactions 33 889-895.

Yang, J., Jaillet, P. and Mahmassani H. 2004. Real-Time multi-vehicle truckload pickup and delivery problems. Transportation Science 38 (2) 135-148.

Yim, D.S. and Linn, R.J. 1993. Push and pull rules for dispatching automated guided vehicles in a flexible manufacturing system, International Journal of Production Research 31 (1) 43-57.

Yu, W. and Egbelu, P.J. 2001. Design of a variable path tandem layout for automated guided vehicle systems, Journal of Manufacturing Systems 20 (5) 305-319.

Zeng, L., Wang, H.P.B. and Jin, S. 1991. Conflict detection of automated guided vehicles: a Petri net approach, International Journal of Production Research 29 (5) 865-879. 


\section{Publications in the Report Series Research* in Management}

\section{ERIM Research Program: "Business Processes, Logistics and Information Systems"}

2004

Smart Pricing: Linking Pricing Decisions with Operational Insights

Moritz Fleischmann, Joseph M. Hall and David F. Pyke

ERS-2004-001-LIS

http://hdl.handle.net/1765/1114

Mobile operators as banks or vice-versa? and: the challenges of Mobile channels for banks

L-F Pau

ERS-2004-015-LIS

http://hdl.handle.net/1765/1163

Simulation-based solution of stochastic mathematical programs with complementarity constraints: Sample-path analysis S. Ilker Birbil, Gül Gürkan and Ovidiu Listeş

ERS-2004-016-LIS

http://hdl.handle.net/1765/1164

Combining economic and social goals in the design of production systems by using ergonomics standards Jan Dul, Henk de Vries, Sandra Verschoof, Wietske Eveleens and Albert Feilzer ERS-2004-020-LIS

http://hdl.handle.net/1765/1200

* A complete overview of the ERIM Report Series Research in Management: https://ep.eur.nl/handle/1765/1

ERIM Research Programs:

LIS Business Processes, Logistics and Information Systems

ORG Organizing for Performance

MKT Marketing

F\&A Finance and Accounting

STR Strategy and Entrepreneurship 